\title{
EL NUEVO NEGOCIO MEDIÁTICO LIDERADO POR NETFLIX: ESTUDIO DEL MODELO Y PROYECCIÓN EN EL MERCADO ESPAÑOL
}

\author{
The new media business concept led by Netflix: \\ a study of the model and its projection into the \\ Spanish market
}

Jessica Izquierdo-Castillo

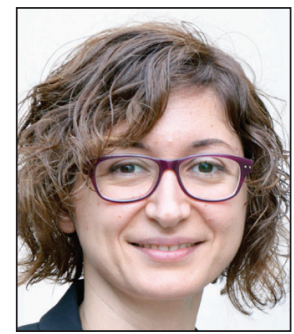

Jessica Izquierdo-Castillo es profesora del Departamento de Ciencias de la Comunicación de la Universitat Jaume I (UJI) de Castellón. Licenciada en comunicación audiovisual por la Universidad de Valencia y en publicidad y relaciones públicas, es doctora en comunicación audiovisual por la UJI. Sus principales líneas de investigación son: la estructura del sistema comunicativo, los modelos de negocio de las empresas comunicativas en el contexto digital y las transformaciones por efecto de la convergencia sobre la distribución de contenidos. Es investigadora en diferentes proyectos I+D, financiados por el Ministerio de Economía y Competitividad del Gobierno de España, la Generalitat Valenciana y la UJI. Ha sido investigadora invitada en la Université Paris 8 (Francia), la Université Grenoble 3 (Francia) y la Universitá Degli Studi di Firenze (Italia).

http://orcid.org/0000-0002-5037-1040

Universitat Jaume I de Castellón Av. Vicente Sos Baynat, s/n. 12071 Castellón, España jizquier@uji.es

\section{Resumen}

El negocio de contenidos mediáticos en internet está liderado por nuevos agentes que vinculan su actividad con la distribución de contenidos. Estos actores trabajan adaptados a las demandas del contexto convergente mediático, y proponen modelos de negocio orientados hacia la satisfacción del usuario. Entre ellos, destaca notablemente el caso de Netflix, por el liderazgo que ejerce en su mercado doméstico, Estados Unidos, así como por su expansión internacional. Se presenta en profundidad el modelo de negocio de Netflix con un caso de estudio que se articula en tres aspectos fundamentales: la fórmula de monetización y configuración de catálogo, la política de relaciones con sus principales públicos (usuarios y proveedores de contenido y de red) y su estrategia de internacionalización. A partir de los resultados, se abre una discusión sobre la proyección de este modelo en el mercado mediático español.

\section{Palabras clave}

Distribución online; Contenido; Contenidos audiovisuales; Medios; Vídeo; Internet; Convergencia; Audiencias; Modelos de negocio; Netflix.

\section{Abstract}

New actors who link their activity to content distribution are leading the business of online media content. These actors adapt their operations to the demands of converging media context, and they propose business models oriented to user satisfaction. Among them, Netflix is notably highlighted for leadership in its home market, the United States, and in its international expansion. This paper presents in detail the Netflix business model with a case study that focuses on three key areas: the catalogue and monetization formula, policy relationships with key audiences (users, content providers and internet providers) and its internationalization strategy. Based on the results, the projection of this model in the Spanish media market can begin to be discussed.

\section{Keywords}

Online distribution; Content; Audiovisual content; Media; Video; Internet; Convergence; Audiences; Business models; Netflix.

Izquierdo-Castillo, Jessica (2015). El nuevo negocio mediático liderado por Netflix: estudio del modelo y proyección en el mercado español". El profesional de la información, v. 24, n. 6, pp. 819-826.

http://dx.doi.org/10.3145/epi.2015.nov.14 


\section{Introducción}

La industria mediática se articula en un entorno convergente que reconfigura el sistema comunicativo e incorpora nuevos líderes (Cunninghan; Silver, 2013) vinculados a modelos disruptores (Cunningham; Silver; McDonnell, 2010) que rompen con el negocio tradicional. El escenario actual es resultado de la combinación de elementos de medios viejos y nuevos, compuesto por productos comunicativos diversos compilados en un único canal (internet).

Esta convergencia no sólo es tecnológica, sino que también incluye una dimensión cultural, un proceso en el que participan los consumidores a través de sus interacciones (Jenkins, 2008). El usuario es actor principal y beneficiario de este cambio, accede a través de un único canal a todo el catálogo de contenido comunicativo y participa en su proceso de creación, difusión y desarrollo.

\section{En su rol de productor Netflix anula una de las premisas de la televisión tradicional}

El nuevo negocio comunicativo es efecto del fenómeno de la disrupción de la distribución de contenidos. Los todavía llamados grandes estudios de Hollywood, que tradicionalmente han concentrado el poder del negocio mediático gracias al control del contenido, ya no ocupan una posición hegemónica. Internet ha constituido un proceso de desintermediación (Iordanova, 2012), que ha conllevado la aparición de una nueva posición de dominio por parte de las plataformas de distribución online de contenidos. Estas plataformas se articulan alrededor de una nueva concepción del negocio mediático, que propone fórmulas enfocadas hacia la satisfacción del usuario. En este grupo se encuentran propuestas diversas:

- la pionera iTunes, que introdujo la opción del pago por unidad (una canción en lugar de un disco, un episodio en lugar de la serie televisiva completa, etc.);

- Hulu, YouTube y Spotify, con acceso abierto a contenido televisivo y musical respectivamente, a cambio de consumo publicitario. Además, las tres plataformas coinciden en combinar esta modalidad con el vídeo bajo demanda.

Esta asociación entre la hegemonía en la industria y el control de contenido mediático que se observa también en el escenario convergente parece repetir el esquema del sistema tradicional. Sin embargo, existen varios factores que lo diferencian:

- el control no lo ostentan los actores directa o indirectamente vinculados en la producción y distribución del contenido (o no necesariamente), que serían los grandes estudios de Hollywood;

- los circuitos de distribución no se circunscriben a un único sector (como el televisivo o el cinematográfico, por ejemplo), sino que se articulan en torno al contenido, que se empaqueta a través de plataformas de distribución (que no se corresponden con los distribuidores anteriores);

- se ha producido un cambio de rol por parte de la audiencia, que pasa a ser activa (Gubbins, 2012) y protagoniza un proceso de empoderamiento del contenido, sintiéndo- se libre para acceder a él (incluso por medios no legales), compartirlo con otros usuarios o transformarlo.

En este nuevo sector de distribución online de contenidos, Netflix destaca con su modelo de vídeo por suscripción. La compañía ha implantado su negocio en casi 80 países y pretende alcanzar en los próximos dos años un total de 200 en todo el mundo. Su modelo de negocio audiovisual se basa en un contenido atractivo y en una fórmula de monetización interesante para el usuario, pero cuenta además con otros factores estratégicos que son clave para entender su progresión. Con esta propuesta, la compañía se ha introducido con éxito en varios países europeos desde 2012 (en España en octubre de 2015).

\section{Objetivos y metodología}

El objetivo principal de este artículo es analizar el caso de estudio de Netflix, atendiendo a tres dimensiones:

- modelo de negocio, a través de la fórmula de monetización;

- política de relaciones con los usuarios y proveedores;

- estrategia de internacionalización.

A partir de aquí se confrontan los resultados del caso de estudio de Netflix con el contexto español.

La elección de este mercado se encuentra motivada por los datos positivos que reflejan su crecimiento, con un consumo cada vez mayor de contenidos online y un elevado grado de equipamiento tecnológico vinculado con la movilidad y la conectividad de alta velocidad. Estos datos presentarían a priori un escenario atractivo para la estrategia expansiva de colonización de la distribución online que desarrolla Netflix, aunque la compañía no lo ha incluido en su primer plan de expansión.

La metodología de estudio de caso utiliza información derivada de la revisión de la bibliografía y de los datos publicados por las propias compañías online, así como por la prensa especializada.

\section{Netflix como paradigma de distribución online: una estrategia en tres puntos}

Netflix surgió como una tienda online de alquiler de vídeo en 1997, con una novedosa fórmula de monetización, basada en la cuota de suscripción para el alquiler de DVDs. En 2007 introdujo el vídeo en streaming, manteniendo el sistema de suscripción mensual, lo que supuso un punto de inflexión en el mercado del vídeo online (Ojer; Capapé, 2013). En ese momento, el sector estaba encabezado por la compañía Apple, que tenía experiencia en la comercialización de música bajo el modelo de paywall (Barr, 2011) de pago por descarga unitaria, en la plataforma iTunes. Cuando introdujo el vídeo en su catálogo, iTunes alcanzó la cifra de un millón de vídeos por semana en 2006 (Ulin, 2012, p. 304) y dominó el mercado hasta 2011, siendo sustituida por Netflix en la primera posición (gráfico 1).

Netflix cerró su primer año de actividad streaming en 2007 con 7,5 millones de suscriptores (Ojer; Capapé, 2013). En 2010 su cuota se situó en el 44\% (gráfico 1), y desde entonces lidera el sector con una tasa de crecimiento interanual 
entre el 35 y el $40 \%$, con ingresos superiores a los 4.500 millones de dólares (gráfico 2).

Tras la buena acogida del incipiente mercado online, la compañía se vio tentada de separar sus líneas de negocio y maximizar beneficios. Por ello anunció en 2011 el lanzamiento de una plataforma asociada, Qwikster, pensada para el vídeo en streaming. De esa forma pretendía crear una nueva marca vinculada con el negocio original de alquiler de vídeo y potenciar Netflix como marca asociada a la distribución online. No obstante, la reacción del público impidió el lanzamiento de la plataforma asociada, pues sólo su anuncio causó la baja de un millón de suscriptores en 2011. Tras la cancelación del proyecto, y una disculpa pública por parte de la compañía, Netflix volvió a su propuesta inicial de tarifa plana de contenidos y actualmente tiene 65 millones de abonados en todo el mundo (Netflix, 2015).

Su éxito puede explicarse con la fórmula de monetización, que permitió a los internautas acceder a un catálogo de contenidos amplios mediante una cuota de suscripción económica (7,99 dólares al mes), lo que suponía una notable ventaja frente a la oferta del resto de plataformas, que cobraban entre 2 y 3 dólares por acceso de 24 horas a una pieza de contenido (Izquierdo-Castillo, 2012). Pero para profundizar en el modelo de Netflix, debe atenderse a otros elementos clave. Entre ellos destacan tres factores:

- modelo de negocio orientado a la demanda;

- política de relaciones con los públicos principales;

- estrategia de internacionalización.

\subsection{Modelo de negocio orientado a la demanda}

Los usuarios cada vez consumen más contenidos online. Esta demanda es más acusada en los jóvenes, que dedican 22 horas a la semana a esta actividad frente a 8,3 horas a la televisión. Este consumo está dividido entre (Defy Media, 2015):

- vídeos de acceso libre (11,3 horas para plataformas como YouTube);

- plataformas de suscripción (10,8 horas semanales para portales como Netflix)

Frente a esta demanda, Netflix ofrece una fórmula ventajosa con una cuota mensual para acceso al catálogo en 3 modalidades:

- contenido en SD disponible en 1 dispositivo (7,99 dólares);

- contenido en HD disponible en 2 dispositivos (8,99 dólares);

- contenido en HD y ultraHD disponible en 4 dispositivos (11,99 dólares).
De esta forma Netflix se aleja del modelo tradicional de suscripción, que fija el precio en función del volumen de contenido y/o de la categorización por calidad (normal frente a premium). Así actúan las televisiones tradicionales en cualquiera de sus canales de distribución (terrestre, satélite, cable), y algunas plataformas online como Hulu Plus (versión de pago de Hulu) o Yomvi (réplica online de Canal Plus). Sin embargo, Netflix focaliza el valor en dos elementos:

- calidad del visionado, distinguiendo SD y HD;

- movilidad y accesibilidad a los contenidos a través de diferente número de dispositivos.

Para un usuario capacitado para acceder a cualquier contenido a través de vías alternativas a estas plataformas (páginas de intercambio de archivos, páginas de descargas no legales, etc.), resulta más atractivo un modelo que le proporcione facilidad y comodidad en el acceso. Esto ofrece una ventaja competitiva frente a las fórmulas alternativas, que suelen presentar elementos molestos como publicidad,

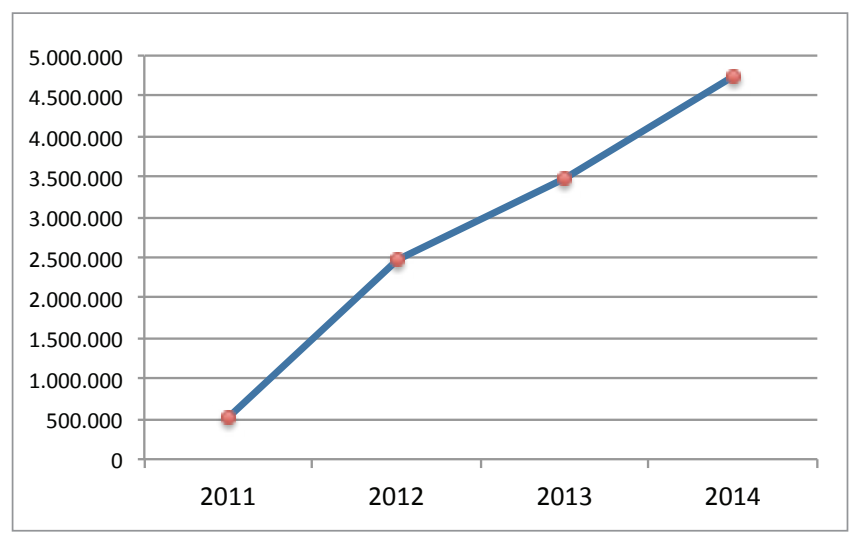

Gráfico 2. Evolución de ingresos del servicio de streaming de Netflix en 2011-2014 (en miles de dólares)

Fuente: Elaboración propia a partir de datos publicados por Netflix 


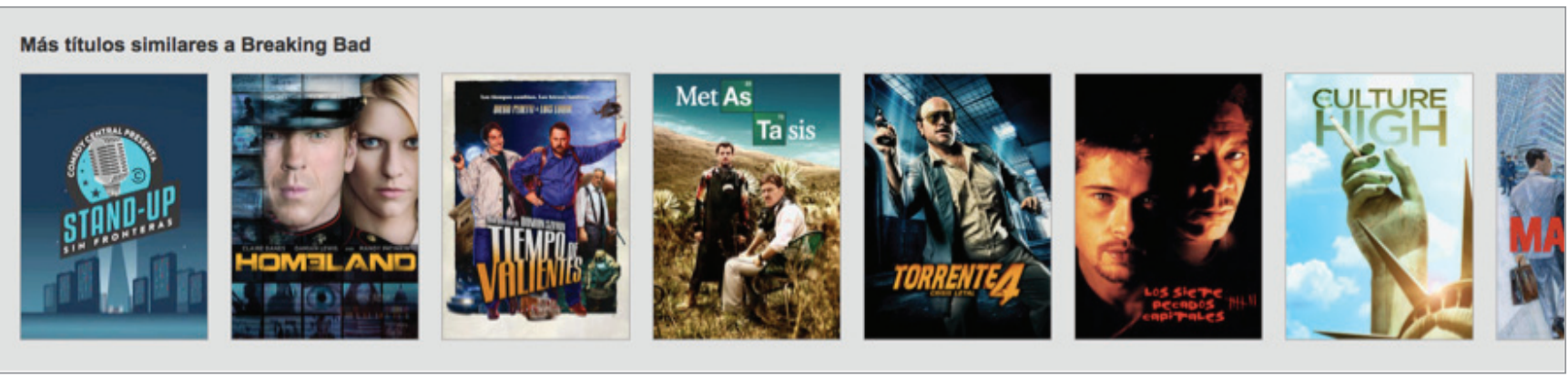

Figura 1. Ejemplo de recomendación basado en contenido consumido Fuente: Netflix

petición de datos, problemas en el streaming o descarga, etc. Por el contrario, los modelos basados en el contenido, suponen un reclamo menor para el usuario que no prioriza el acceso al contenido, sino la calidad de ese acceso.

No obstante, Netflix también considera el contenido como elemento estratégico de su modelo de negocio. A las películas y series adquiridas a terceros, la compañía suma producciones donde su participación es directa (House of cards, Marco Polo, Orange is the new black). De esta forma, Netflix replica el papel que las networks han desempeñado durante los últimos años, como principales impulsoras de la creación de los contenidos audiovisuales (Cunninghan; Silver, 2013, p. 89) y entra en competencia directa, además de con otras plataformas, con cadenas tradicionales como la HBO. Esta cadena televisiva de pago estadounidense tiene su propio servicio de streaming del canal a través de $H B O$ Go y, desde marzo de 2015, también un servicio premium (sin necesidad de suscripción al canal de pago) con HBO Now a través de Apple TV (14,99 dólares al mes). Por otro lado, Netflix también está enfrentada con Amazon y su servicio Amazon Prime Instant Video, que funciona como elemento integrado en el paquete Amazon Prime (99 dólares anuales), y que se calcula cuenta con 14,5 millones de suscriptores (Greene, 2015).

Proporciona una oportunidad de negocio a productores independientes $y$ otros productos con escasa visibilidad en circuitos comerciales

La disrupción de Netflix en el mercado de contenidos también afecta a la premisa del negocio televisivo y su contenido seriado. En su rol de productor, la compañía ha sido la primera y única en ofrecer las temporadas completas de sus series en el momento de su estreno. De esta forma anula una de las premisas de la televisión tradicional, que consiste en la serialidad de los contenidos, para favorecer la fidelización de la audiencia y obtener de esa forma una estabilidad en los ingresos publicitarios o de suscripción. La compañía afirma que el estreno de House of cards supuso un incremento de 3 millones de abonados (Kaiser, 2013), lo que se traduce en importantes ingresos, que posibilitan la producción de la serie, con un presupuesto medio de 3,8 millones de dólares por capítulo (Greenfield, 2013).
No obstante, con independencia de los resultados, esta estrategia le permite crear marca y reforzar su posición de liderazgo en el mercado, que redunda en un incremento de abonados. Al mismo tiempo, le dota de un fuerte posicionamiento en la mente del espectador, que refuerza la imagen de marca de la plataforma como referente del mercado de distribución online y plantea un importante reto al resto de plataformas, al romper la pauta de periodicidad seriada en el estreno de contenidos.

\subsection{Política de relaciones con los públicos principales}

\subsubsection{Escrutinio de los usuarios}

El modelo de Netflix se enfoca en la experiencia del usuario y en la maximización de los recursos. Para ello ha desarrollado un complejo software de procesamiento de datos, que consigue un sofisticado sistema de recomendación focalizado en el diseño, construcción y optimización de los contenidos. Netflix dedica aproximadamente 300 trabajadores e invierte 150 millones de dólares anuales en investigación de algoritmos, interfaces de usuario, plataformas de recomendaciones y todo lo relacionado con los metadatos de la plataforma (Kuburas, 2014).

Los beneficios de esta inversión son múltiples:

- ofrece información relevante para la recomendación de productos, que se convierte en un valor añadido del servicio (figura 1);

- permite mejorar el diseño del catálogo, optimizando los recursos, por ejemplo limitando la compra de contenidos infantiles, pues es un público permisivo con la continua repetición de un programa (Rogowsky, 2014), que se adapta a las posibilidades y demandas de cada mercado en el que opera.

de esta forma, los sistemas de automatización permiten evaluar el contenido antes de adquirirlo para la plataforma, incluso recomendar actores para la interpretación de papeles en una producción propia [Hunt, en Kuburas (2014)] o rediseñar el catálogo en función de la demanda; da visibilidad a contenidos menos comerciales, que los usuarios se animan a consumir alentados por las recomendaciones que reciben. Esto proporciona una oportunidad de negocio a productores independientes y líneas de productos de los estudios tradicionales con escasa visibilidad en circuitos comerciales generales, lo que redunda en una buena posición para Netflix frente a la negociación con los proveedores de contenido. 


\subsubsection{Cambio de rol con los proveedores de contenido}

El catálogo de Netflix se compone de películas, series de televisión y documentales. Aunque en los últimos años ha incrementado el número de producciones propias, todavía se puede considerar una estrategia en prueba, ya que el grueso de su catálogo proviene de contenido adquirido por concesión. Entre los acuerdos más significativos de la compañía se cuentan los alcanzados con Disney para las diferentes líneas de la compañía, como Marvel o Pixar. También los acuerdos de concesión con Fox Television o con CBS Studios.

El modelo de Netflix se enfoca en la experiencia del usuario y en la maximización de los recursos

Las negociaciones que Netflix debe articular con los proveedores de contenido aumentan de complejidad a medida que el negocio crece y se expande. Los acuerdos no sólo deben realizarse atendiendo al mercado doméstico y su estructura (concesiones con otros operadores del mercado), sino a las diferentes negociaciones que los proveedores de contenido hayan adquirido en cada uno de los mercados externos donde Netflix opera.

La gran base de suscriptores le proporciona capacidad financiera para pujar por los contenidos en las negociaciones frente a otros distribuidores. Netflix ha pasado de ser una ventana más en la cadena de explotación comercial del contenido a ocupar una posición de referencia. Su interés por un contenido puede determinar su renovación por parte de la productora o hacer aumentar su valor en el mercado de las pujas por la concesión de derechos. En este sentido, una de las principales necesidades de Netflix en las negociacio- nes con los proveedores de contenido, junto con la obtención de derechos en exclusiva, es la flexibilidad en la contratación, que le permita modificar su catálogo en función del consumo de sus suscriptores.

\subsubsection{Demandas para los proveedores de servicio de in- ternet (PSI)}

Otra clave es el fomento de las relaciones con los proveedores de servicio de internet (PSI). El modelo de Netflix, al igual que el resto de plataformas online, depende del tráfico de datos por internet. La relación con los PSI es fundamental para garantizar el correcto servicio de datos. Una banda ancha ralentizada o un mal servicio de tráfico de datos puede suponer pérdidas cuantiosas para Netflix. Los datos de consumo de internet reflejan una gran demanda de vídeo online. En Norteamérica por ejemplo, Netflix es responsable del $34,9 \%$ del tráfico de datos de bajada (Sandvine, 2014).

La posición predominante de Netflix le proporciona ventaja frente al problema de la saturación del tráfico en internet. Ante el debate sobre la gestión del volumen de datos, la empresa ha abogado por la neutralidad de la red, pues cualquier medida selectiva repercutiría en costes para su actividad. De hecho, una de las amenazas económicas para estas plataformas de vídeo bajo demanda son las tarifas que los PSI pretenden gravar sobre los prestadores que mayor volumen de datos consumen, pero también la gran dependencia del modelo de streaming de los servicios de alojamiento y tráfico de contenidos.

Netflix también tiene una delicada relación con Amazon, a través del servicio de Amazon Web Services (AWS). Amazon provee a Netflix de una plataforma informática de distribución basada en la "nube" (espacio virtual de almacenamiento). De esta forma, recibe de AWS soporte para el procesamiento de datos, almacenamiento y otros servicios. Esta relación crea una dependencia respecto de Amazon, que a

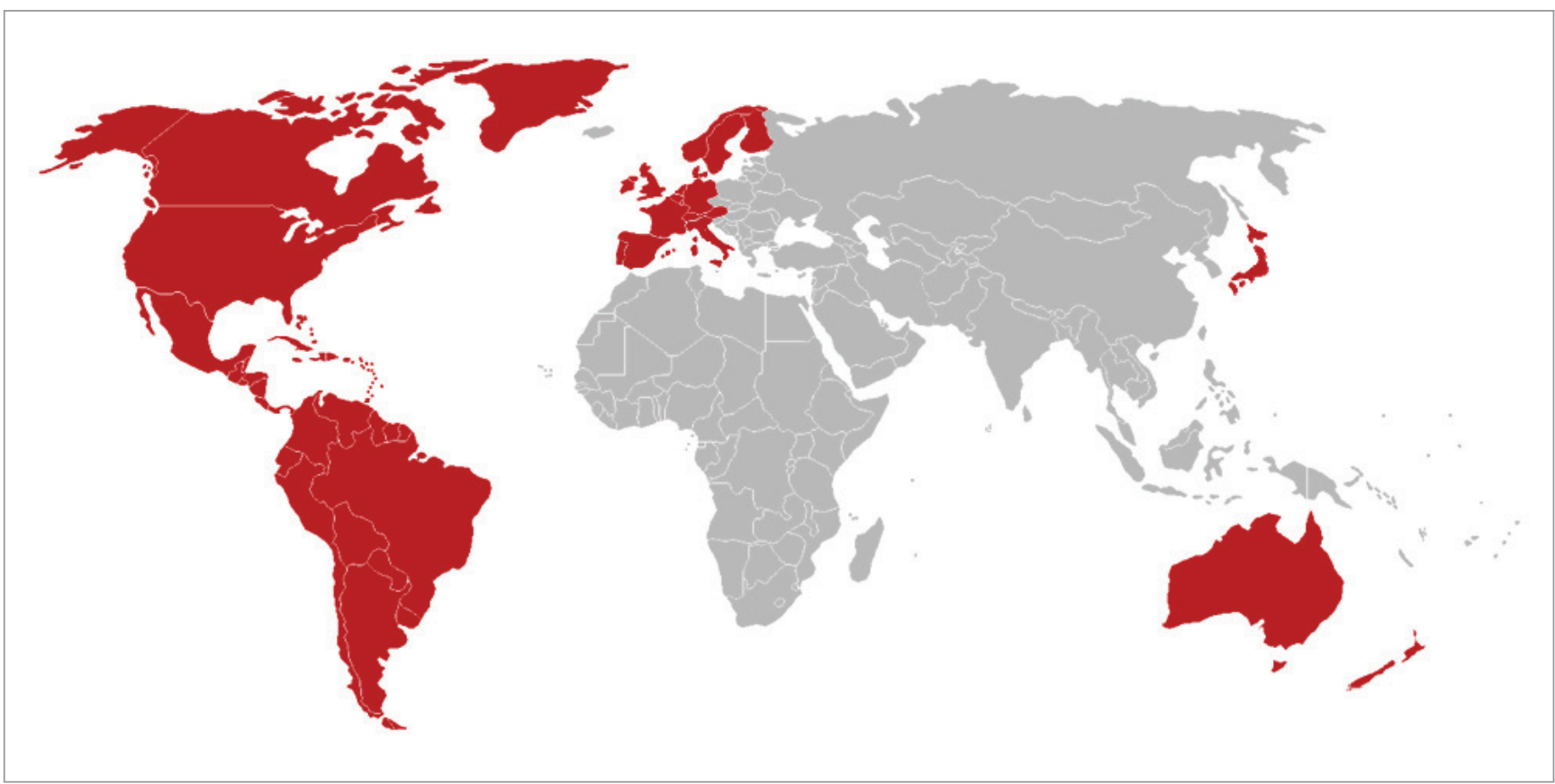

Figura 2. Presencia mundial de Netflix (sept. 2015)

Fuente: netflix.com 
su vez tiene sus propios intereses en el mismo negocio, con su filial Amazon Instant Video (Netflix, 2015).

En Europa la neutralidad de red es objeto de debate continuo, por el enfrentamiento entre el Comisionado de Telecomunicaciones de la UE y los operadores de telecomunicaciones. En abril de 2014 el Parlamento Europeo votó unas normas para evitar que los operadores bloqueen o restrinjan contenido con el objeto de gestionar el tráfico de datos. En EUA el debate quedó zanjado en febrero de 2015, con la aprobación por parte de la Comisión Federal de Telecomunicaciones del reconocimiento de internet como un servicio básico, lo que garantiza la neutralidad de red.

\section{El interés de Netflix por un contenido} puede determinar su renovación o aumentar su valor en el mercado

\subsection{Estrategia de internacionalización}

Tradicionalmente los mercados internacionales han supuesto una importante ventana de negocio para el sector audiovisual estadounidense. En cifras Europa representa el 40,4\% de la cuota de explotación internacional de las principales compañías cinematográficas y unos ingresos de 10,6 billones de dólares (MPAA, 2015). En un escenario convergente y global, este proceso de internacionalización es más rápido y flexible.

Netflix comenzó su internacionalización en 2010, con su entrada en Canadá. En 2011 reforzó su política de crecimiento internacional tras "un problema financiero" (Cunninghan; Silver, 2013, p. 88) derivado del intento fallido de su filial. Entonces lanzó su servicio en América Latina y preparó su entrada en Europa para 2012. Primero se introdujo en el Reino Unido e Irlanda, y a finales de año en Suecia, Dinamarca, Noruega y Finlandia. El proceso continuó en 2014 con Austria, Bélgica, Francia, Alemania, Luxemburgo y Suiza. En 2015 Netflix ha ampliado su cobertura con Japón, Nueva Zelanda, España, Italia y Portugal (figura 2).

En términos económicos, los ingresos de Netflix experimentaron un crecimiento importante en un breve período, pasando de los 300 millones de dólares en 2012 a 1.481 millones de dólares en 2014 (Netflix, 2015). Actualmente, el $36,3 \%$ de sus abonados pertenecen a su línea internacional (gráfico 3).

El resultado de esta estrategia de tres puntos ha convertido a Netflix en una plataforma de distribución de referencia en el mercado mundial. Pero además de esta función, ha intensificado notablemente su rol como productora de contenidos, participando en la creación de series de televisión y, desde 2015, también de largometrajes (Safo, 2015). De esta forma refuerza su posición de dominio y marca las pautas para el cambio de modelo. En televisión, este cambio se ilustra con la transgresión de la serialidad. En cine, la compañía no diferencia entre su ventana y la sala de cine, estrenando las películas de manera simultánea. Con estos movimientos la compañía marca las pautas de un nuevo negocio que expande por todo el mundo.

\section{Proyección en el mercado español}

El dominio de Netflix en el panorama mundial de distribución de contenido en streaming le ubica al mismo tiempo en una posición vulnerable frente a factores que amenazan su modelo de negocio. A través de la identificación que la propia compañía realiza de estos factores se puede establecer una confrontación con el contexto español. De esta forma se ejemplifica con un caso concreto los elementos que pueden frenar el avance de Netflix o motivar su falta inicial de interés por mercados específicos como el español (Asián, 2014; Europa Press, 2015).

En primer lugar destaca la necesidad de mantener y aumentar la base de suscriptores. En España el mercado de pago para contenidos televisivo y cinematográfico es pequeño, con 4,8 millones de abonados entre las plataformas de satélite, cable, IP, móviles y TDT de pago (CNMC, 2014). Por el contrario existe un elevado consumo de vídeo online. Los datos revelan que el $85,5 \%$ de las personas que se conectan a internet lo hacen para consumir medios comunicativos. La contradicción entre ambos parámetros se explica por la presencia de la piratería o consumo de páginas no legales. Según datos del Observatorio de Piratería y Hábitos de Consumo de Contenidos Digitales, el 51\% de los usuarios descarga contenidos protegidos por derechos de propiedad intelectual. Esta dinámica dificulta la consolidación de un modelo de pago, incluso con tarifas atractivas como las de Yomvi Play (plataforma de

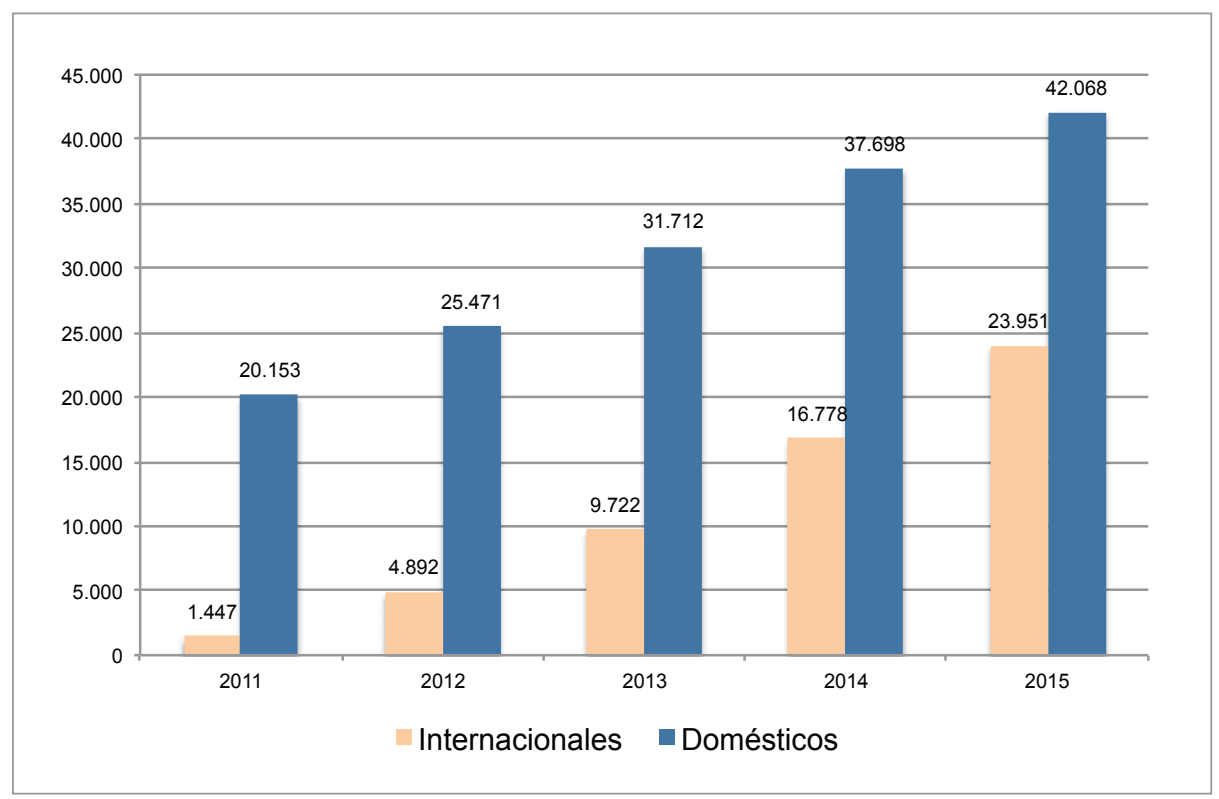

Gráfico 3. Evolución de los abonados domésticos e internacionales de Netflix en 2011-2015 (datos a septiembre de 2015)

Fuente: Netflix 
suscripción online que no exige estar abonado al canal) por 6 euros mensuales, o Wuaki por 6,99 euros. Netflix es una opción menos económica, con su nueva tarifa incrementada un 12,5\% en 2014 (pasando de 7,99 dólares a 8,99 dólares), que supondría una tarifa de 7,99 euros al mes.

\section{No existe un modelo de negocio líder sino modelos flexibles que encuentran posi- ciones de dominio de forma puntual}

En segundo lugar el auge de la competencia es notable y se manifiesta con diferentes modelos de negocio: otras plataformas de suscripción de vídeo en streaming, las plataformas online de los operadores tradicionales, los proveedores online de películas y contenido televisivo (incluidos los ilegales) y los servicios de alquiler de DVD. De este conjunto destacan por un lado las webs de acceso ilegal a contenidos, que plantean una competencia difícil de igualar pues ofrecen un acceso gratuito a un amplio catálogo. Pero también destaca la competencia de los operadores tradicionales, que cuentan con una serie de ventajas sobre su modelo:

- años de experiencia en el sector del vídeo;

- gran base de clientes;

- fuerte reconocimiento de marca;

- recursos financieros.

Esto les otorga ventaja frente a las negociaciones con los principales proveedores de contenido, que mantienen por el momento su posición hegemónica en la distribución cinematográfica, también en el escenario digital (IzquierdoCastillo, 2010). En España este lugar lo ocupan Canal Plus a través de la televisión por satélite y desde 2014 de Yomvi Play, y Telefónica con el servicio Movistar Televisión y desde 2014 Movistar Series (sólo para abonados al paquete de servicio Movistar Fusión, con un suplemento de 7 euros al mes). Ambos paquetes de oferta controlados por Telefónica, que obtiene una posición estratégica en el sector audiovisual de pago por internet.

En tercer lugar destaca la dependencia de Netflix de la estabilidad en las políticas relacionadas con su negocio, desde las políticas de diversidad cultural hasta las de protección de datos. Las leyes españolas de protección intelectual pueden ser un factor disuasorio, como la propia compañía reconoció en 2011, cuando manifestó que los "abusivos derechos de autor en España representarían un coste de 2 a 3 veces mayor que esos mismos derechos en otros países de nuestro entorno, como Francia o Alemania" (Asian, 2014). Esta situación no se resuelve con la última Ley de propiedad intelectual, en vigor desde 2015, que amplía la persecución de la distribución ilegal de contenidos a las páginas de internet que incluyen enlaces a otras webs que albergan contenidos, sean o no legales. De esta forma la $L P I$ refuerza el camino iniciado con la Ley de economía sostenible de 2011 (más conocida como Ley Sinde) y penalizan al usuario final y vulneran sus libertades (Sarikakis; Rodríguez-Amat, 2014, p. 5), al mismo tiempo que protegen los intereses de modelos económicos basados en estructuras tradicionales.

\section{Conclusión}

Netflix marca la pauta del nuevo negocio mediático como líder del sector de distribución online de contenidos. Este modelo no es estable ni fijo, sino que se encuentra sometido a la indefinición que caracteriza al escenario convergente. Esto se traduce en una continua adaptación de las propuestas de las empresas, incluso de las dominantes del sector.

El propio modelo de suscripción de vídeo bajo demanda de Netflix es en sí mismo una evolución de su negocio original, el alquiler online de DVD, y a su vez ha evolucionado hacia la creación de una plataforma que se comporta además como un nuevo estudio de producción. Esto ocurre también con su competencia, que experimenta con la hibridación de modelos. Los ejemplos se observan a escala global en $H B O$, Amazon o Hulu, pero también en contexto más pequeños, en Yomvi Play.

Los tres elementos combinados para el éxito en la distribución online son: catálogo amplio; tarifa económica y sin permanencia; y servicio enfocado a calidad de la imagen y accesibilidad multiplataforma

Por tanto no existe un modelo de negocio líder, sino modelos flexibles que encuentran posiciones de dominio de forma puntual. Estos modelos se encuentran en constante evolución. Además son altamente sensibles a la aprobación de la demanda, que cada vez es más exigente. Por el contrario sí pueden establecerse las claves estratégicas que configuran la base de los nuevos modelos mediáticos, que se resumen en tres elementos interconectados:

- catálogo amplio y variado, que incluya contenido premium en su oferta de base;

- tarifa económica y sin permanencia que permita acceso ilimitado al catálogo;

- servicio enfocado a la calidad de la imagen, la facilidad en la navegación, la búsqueda de contenidos afines y la accesibilidad a través de múltiples dispositivos.

Con estos elementos combinados cualquier plataforma es potencialmente un distribuidor exitoso. En el contexto español existen plataformas que podrían liderar ese mercado, que demuestra un elevado interés por el vídeo online. No obstante se enfrentan a barreras que impiden su crecimiento, como el tamaño limitado del mercado y una escasa cultura del pago por consumo de contenido. Esto no proporciona una masa crítica suficiente para sostener los costes de adquisición, lo que representa una ventaja competitiva para las compañías que puedan aprovechar economías de escala derivadas de una estrategia de expansión, como es el caso de Netflix. Sin embargo, el retraso en el mercado español ha permitido la organización de la competencia, entre la que destaca Telefónica, que tiene una posición estratégica en el mercado de los operadores de telecomunicación. 


\section{Nota}

1. Este artículo se enmarca dentro del proyecto de investigación (P1·1A2014-05), dirigido por el Dr. Javier Marzal-Felici y financiado por la Universitat Jaume I de Castellón.

\section{Bibliografía}

Asián, Arantxa (2014). "Netflix planea su llegada a España para el último trimestre de 2015". Muycomputerpro.com, 30 de julio.

http://www.muycomputerpro.com/2014/07/30/netflix-espana

Barr, Trevor (2011). "Television's newcomers: Netflix, Apple, Google and Facebook". Telecommunications journal of Australia, v. 61, n. 4, pp. 60.1-60.10.

http://researchbank.swinburne.edu.au/vital/access/ services/Download/swin:25077/SOURCE2

CNMC (2014). Nota trimestral del sector audiovisual. $3^{r}$ trimestre 2014. Comisión Nacional de los Mercados y la Competencia. http://goo.gl/DZm5FR

Cunningham, Stuart; Silver, Jon (2013). Screen distribution and the new King Kongs of the online world. New York: Palgrave Pivot. ISBN: 9781137326461

Cunningham, Stuart; Silver, Jon; McDonnell, John (2010). "Rates of change: Online distribution as disruptive technology in the film industry". Media international Australia incorporating culture and policy, n. 136 (August), pp. 119-132. http://eprints.qut.edu.au/39387

Cunninghan, Tod (2015). “Inside Amazon's offensive: How the streaming giant plans to win the game". Thewarap.com, 20 de enero.

http://www.thewrap.com/the-amazon-offensive-digitalgiants-movie-push-challenges-studios

Defy Media (2015). Acumen report. Constant content. Acumen Insights Portal, March.

http://www.defymedia.com/acumen/acumen-reportconstant-content

Europa Press/Madrid (2015). “Netflix llegará en septiembre de 2015 a España”. El periódico.com, 11 de marzo. http://goo.gl/YGK8ng

Greene, Jay (2015). “Amazon's streaming-video service making gains on Netflix". Seattletimes.com, 11 de marzo. http://www.seattletimes.com/business/amazon/amazonsstreaming-video-service-making-gains-on-netflix

Greenfield, Rebecca (2013). "The economics of Netflix's $\$ 100$ million new show". Thewire.com, Febr. $1^{\text {st }}$.

http://www. thewire.com/technology/2013/02/economicsnetflixs-100-million-new-show/61692

Gubbins, Michael (2012). "Digital revolution. Active audiences and fragmented consumption". En: Iordanova, Dina; Cunninghan, Stuart (eds.). Digital disruption: Cinema moves on-line. St. Andrews: St. Andrews Film Studies. ISBN: 9780 956373076

Iordanova, Dina (2012). "Digital disruption: Technological innovation and global film circulation". En: Iordanova, Dina; Cunninghan, Stuart (eds.). Digital disruption: Cinema moves on-line. St. Andrews: St. Andrews Film Studies. ISBN: 9780 956373076

Izquierdo-Castillo, Jéssica (2010). "Distribución en el contexto cinematográfico: la consolidación de la hegemoníaa digital de Hollywood". Área abierta, n. 27.

http://revistas.ucm.es/index.php/ARAB/article/view/ ARAB1010330002A

Izquierdo-Castillo, Jéssica (2012). "Distribución online de contenidos audiovisuales: análisis de 3 modelos de negocio". El profesional de la información, v. 21, n. 4, pp. 385-390. http://repositori.uji.es/xmlui/bitstream/handle/10234/70260/53479. pdf?sequence $=3$ http://dx.doi.org/10.3145/epi.2012.jul.09

Jenkins, Henry (2008). Convergence culture: la cultura de la convergencia de los medios de comunicación. Barcelona: Ediciones Paidós. ISBN: 9788449321535 https://stbngtrrz.files.wordpress.com/2012/10/jenkinshenry-convergence-culture.pdf

Kaiser, Tiffany (2013). "Netflix says House of cards is the reason for subscriber growth". Dailytech.com, Abril 23 $3^{\text {rd }}$. http://www.dailytech.com/Netflix+Says+House+of+Cards+i stthe+Reason+for+Subscriber+Growth/article30404.htm

Kuburas, Melita (2014). “Netflix's data engine worth \$500M a year". Streamdaily.tv, October $10^{\text {th }}$.

http://streamdaily.tv/2014/10/10/netflixs-data-engineworth-500m-a-year

Motion Pictures Association of America (MPAA) (2015). 2014 theatrical market statistics.

http://www.mpaa.org/research-and-reports

Netflix (2015). Q2 15 letter to stakeholders.

http://ir.netflix.com/results.cfm

Ojer, Teresa; Capapé, Elena (2013). “Netflix: A new business model in the distribution of audiovisual content". Journalism and mass communication, v. 3, n. 9, pp. 575-584. http://www.davidpublishing.com/DownLoad/?id=14784

Rogowsky, Mark (2014). "How are the negotiating dynamics changing between Netflix and the movie/TV studios?". Forbes.com, Jan. $10^{\text {th }}$. http://goo.gl/8EVtgy

Safo, Nova (2015). "Netflix becomes a movie studio". Marketplace.com, Jan. $16^{\text {th }}$.

http://www.marketplace.org/topics/tech/netflix-becomesmovie-studio

Sandvine (2014). Global internet phenomena report 2H 2014. http://www.sandvine.com

Sarikakis, Katharine; Rodríguez-Amat, Joan-Ramon (2014). "Intellectual property law change and process: The case of Spanish Ley Sinde as policy laundering". First Monday, v. 19, n. 3, March $3^{\text {rd }}$.

http://firstmonday.org/ojs/index.php/fm/article/view/4854 http://dx.doi.org/10.5210/fm.v19i3.4854

Ulin, Jeffrey C. (2013). The business of media distibution. Monetizing films, TV ad video content in an online world. Oxford: Elsevier Focal Press. ISBN: 9780240812007 